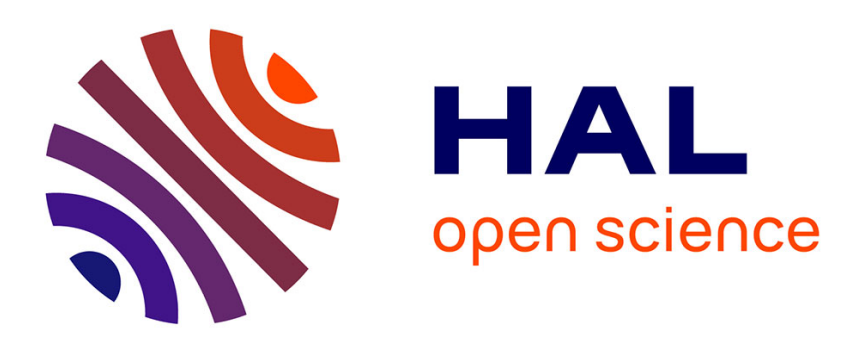

\title{
Suivi de l'endommagement dans les structures en composites par une analyse optique in-situ
}

Nicolas Revest, Jacques Renard, Alain Thionnet, Laurent Boulay, Philippe Castaing

\section{- To cite this version:}

Nicolas Revest, Jacques Renard, Alain Thionnet, Laurent Boulay, Philippe Castaing. Suivi de l'endommagement dans les structures en composites par une analyse optique in-situ. Revue de Métallurgie, 2010, 107, pp.15-20. 10.1051/metal/2010007 . hal-00481356

\section{HAL Id: hal-00481356 \\ https://hal-mines-paristech.archives-ouvertes.fr/hal-00481356}

Submitted on 27 Mar 2012

HAL is a multi-disciplinary open access archive for the deposit and dissemination of scientific research documents, whether they are published or not. The documents may come from teaching and research institutions in France or abroad, or from public or private research centers.
L'archive ouverte pluridisciplinaire HAL, est destinée au dépôt et à la diffusion de documents scientifiques de niveau recherche, publiés ou non, émanant des établissements d'enseignement et de recherche français ou étrangers, des laboratoires publics ou privés. 


\title{
Suivi de l'endommagement dans les structures en composites par une analyse optique in-situ ${ }^{\star}$
}

\author{
N. Revest ${ }^{1}$, J. Renard ${ }^{1}$, A. Thionnet ${ }^{1,2}$, L. Boulay ${ }^{3}$ et P. Castaing ${ }^{3}$ \\ 1 Centre des Matériaux, MinesParisTech, Évry, France \\ 2 Université de Bourgogne, Mirande, Dijon, France \\ 3 CETIM, Nantes, France
}

\begin{abstract}
Résumé - Ce travail présente une technique de détection et de suivi de l'endommagement dans les structures en matériaux composites. Les mécanismes d'endommagement dans ces matériaux sont complexes. Dès lors que l'on souhaite modéliser le comportement d'une structure, il est important de comprendre comment celle-ci s'endommage. La méthode optique d'observation in-situ proposée est appliquée au cas d'une plaque trouée.
\end{abstract}

Abstract - Monitoring of damage in composite structures by optical analysis insitu. This work presents a technique for detecting and monitoring damage in composite structures. The damage mechanisms in these materials are complex. Since we want to model the behavior of a structure, it is important to understand how it gets damaged. The optical method of observation in-situ proposed is applied to the case of a perforated plate.

- a forte anisotropie couplée à l'hétérogénéité de la microstructure des matériaux composites fibres/résine, font que les phénomènes d'endommagement rencontrés dans ces matériaux sont complexes. En effet, la ruine des matériaux composites ne résulte pas d'un mécanisme unique mais est la conséquence de l'accumulation de plusieurs modes de dégradation (décohésion fibre/matrice, fissuration, délaminage, ruptures de fibres...). La connaissance et la compréhension de tous ces mécanismes prennent toute leur importance dès lors que l'on souhaite dimensionner une structure. Il est alors nécessaire de capitaliser des données expérimentales qui permettront d'écrire et d'alimenter un modèle de comportement. On comprend alors l'importance de pouvoir, non seulement détecter l'endommagement mais également de pouvoir identifier les différents phénomènes et leurs interactions.

De nos jours, il existe diverses méthodes de détection de l'endommagement dans les matériaux composites. Parmi les plus couramment utilisées on trouve les méthodes basées sur l'émission acoustique [1] qui consistent à analyser la libération d'énergie sous forme d'ondes élastiques au sein du matériau lorsque ce dernier s'endommage. Toutefois, aucune étude ne démontre clairement que l'on est capable de distinguer, ni de localiser les différents types d'endommagement dans le cas où plusieurs dégradations sont présentes simultanément. Enfin, les ondes acoustiques sont créées par l'apparition du dommage si bien que cette technique ne permet pas de détecter l'endommagement initialement présent au sein du matériau. On peut citer également les méthodes fondées sur la technologie des rayonnements $X$. Le principal inconvénient de cette méthode est l'impossibilité de faire des observations in-situ en cours d'essai. La micro-tomographie [2] permet l'observation en cours d'essais et permet d'avoir accès à la géométrie en trois dimensions des défauts, cependant la petite taille de l'échantillon observé devient rapidement limitante. Il existe encore d'autres techniques telles que l'utilisation de fibres optiques [3], les ultrasons [4], les micro-ondes [5], les mesures de courants [6], la thermographie infrarouge [7]. Cependant, la plupart de ces techniques sont lourdes à mettre en œuvre. Aussi préfèrerons-nous une technique plus légère basée sur une méthode optique de détection et suivi de l'endommagement in-situ. 


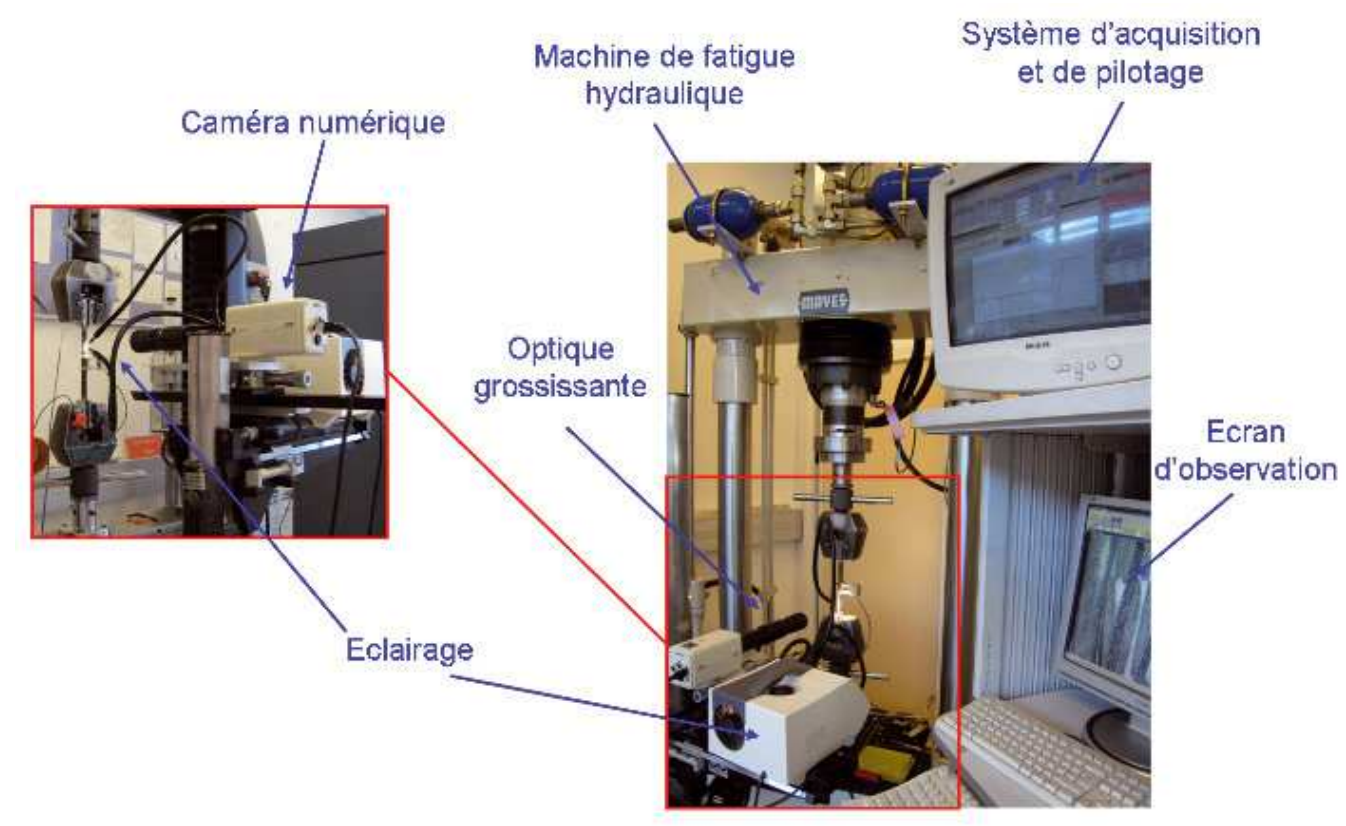

Fig. 1. Dispositif expérimental de suivi in-situ de l'endommagement.

Fig. 1. Experimental setup of in-situ monitoring of damage.

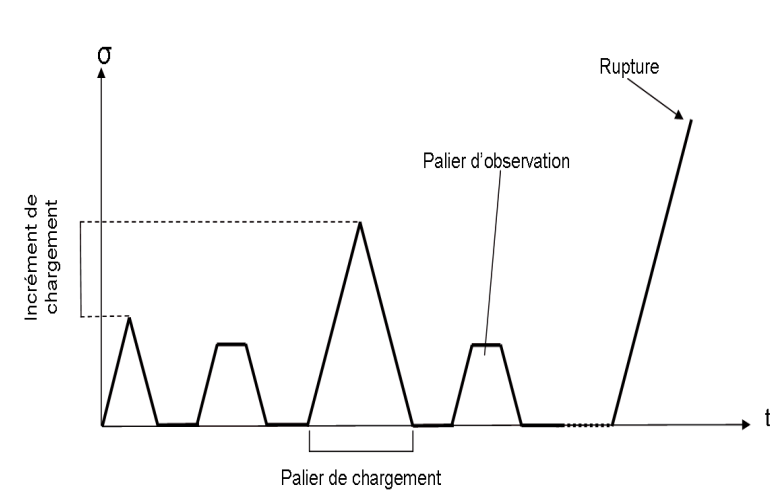

(a)

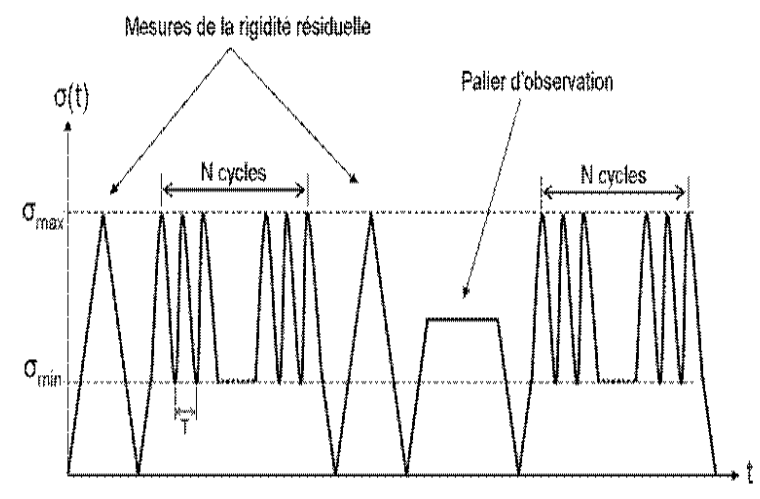

(b)

Fig. 2. Chargements (a) Quasi-Statiques et (b) Cycliques utilisés pour l'analyse de l'endommagement.

Fig. 2. Quasi-static (a) and cyclic loadings (b) used for damage analysis.

\section{Une méthode optique de suivi de I'endommagement in-situ}

La technique consiste à observer et à photographier une zone de l'éprouvette (dans notre cas le bord libre de l'éprouvette) à l'aide d'une caméra montée sur une colonne autorisant des déplacements dans les trois directions permettant de balayer tout le champ de l'éprouvette. La caméra CCD couplée à un zoom optique grossissant jusqu'à 12 fois permet d'accéder aux détails de la microstructure. Le bord libre de l'éprouvette doit être poli au préalable pour faciliter l'observation (Fig. 1).

Outre la simplicité de mise en œuvre, l'avantage de cette méthode est d'éviter le démontage de l'éprouvette après chaque sollicitation et donc de limiter le nombre d'opérations. De plus l'observation peut se faire sous charge ce qui facilite la détection de l'endommagement. Enfin, les éprouvettes testées sont équipées d'un extensomètre afin de mesurer le déplacement longitudinal et d'avoir accès à la rigidité longitudinale du stratifié.

Dans le cas quasi-statique, afin d'avoir une meilleure précision et de simplifier la détection de l'endommagement, l'observation est faite en cours d'essais de charge/décharge avec augmentation de la charge à chaque palier (Fig. 2a). Chaque palier se décompose en deux étapes : la première permet d'étudier le comportement du matériau et de créer l'endommagement et la seconde permet l'observation et les prises de vues. Suivant le même principe les chargements 


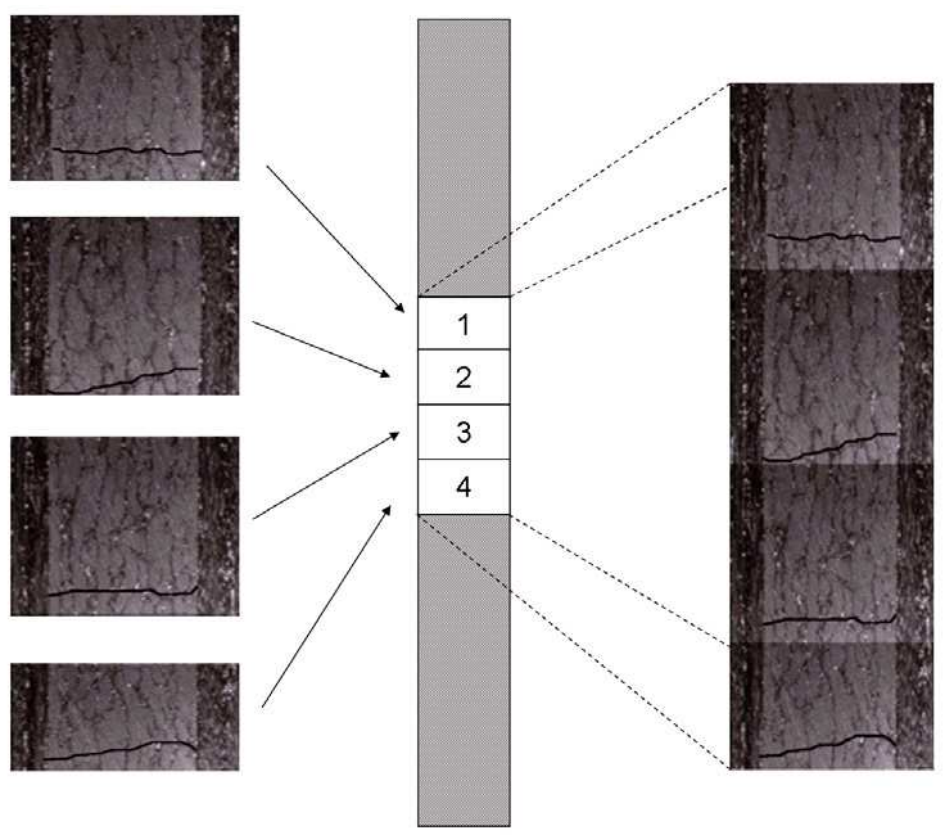

Fig. 3. Reconstruction du bord libre d'une éprouvette fissurée.

Fig. 3. Free edge reconstruction of a cracked sample.
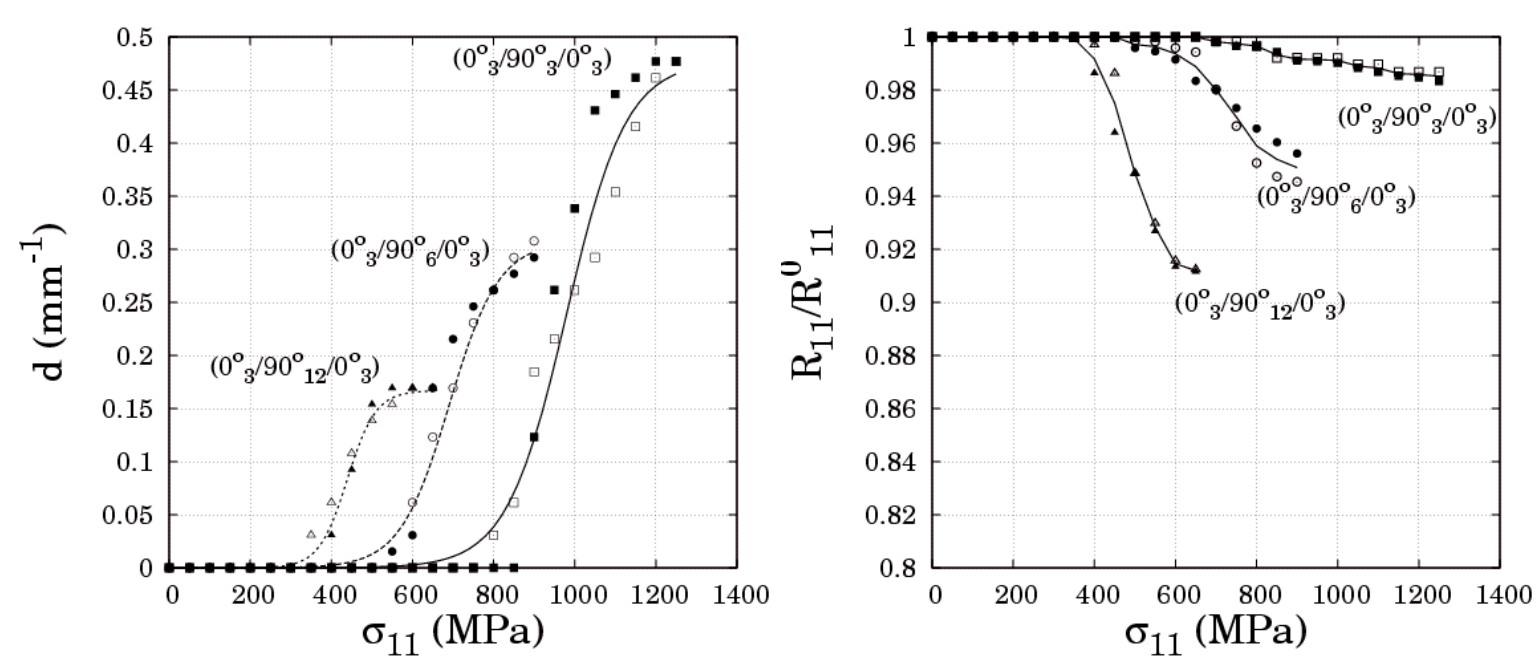

Fig. 4. Évolution de la densité de fissures dans la couche à $90^{\circ}$ et de la rigidité résiduelle pour les séquences $\left(0_{3}^{\circ} / 90_{n=3,6,12}^{\circ} / 0_{3}^{\circ}\right)$ sous chargements quasi-statiques.

Fig. 4. Evolution of cracks density in the $90^{\circ}$ layer and the residual stiffness of $\left(0_{3}^{\circ} / 90_{n=3,6,12}^{\circ} / 0_{3}^{\circ}\right)$ laminates under static loadings.

cycliques se décomposent en trois étapes : un palier de charge/décharge pour mesurer la rigidité longitudinale, l'application de $\mathrm{N}$ cycles de sollicitations et un palier d'observation (Fig. 2b).

À partir des différentes photographies du champ de l'éprouvette nous pouvons reconstruire une image de la tranche de l'échantillon (Fig. 3). L'analyse de l'image obtenue nous renseignera alors sur la présence d'un ou plusieurs types d'endommagement, sur leur position, leur géométrie et nous pourrons également les quantifier.

La méthode présentée ici a comme principal avantage de permettre une visualisation de l'endommagement et ainsi d'acquérir des informations à la fois quantitatives et qualitatives. En outre, cette méthode permet d'identifier les différents mécanismes d'endommagement et d'établir leur chronologie d'apparition, leurs interactions mais elle permet également de quantifier l'état d'endommagement du matériau.

Sous l'effet d'un chargement de traction monotone, les fissures se multiplient en se propageant quasi-instantanément dans l'épaisseur et la largeur du pli jusqu'à atteindre un état de saturation (Fig. 4).

Sous l'effet d'un chargement cyclique le phénomène est comparable. La différence 

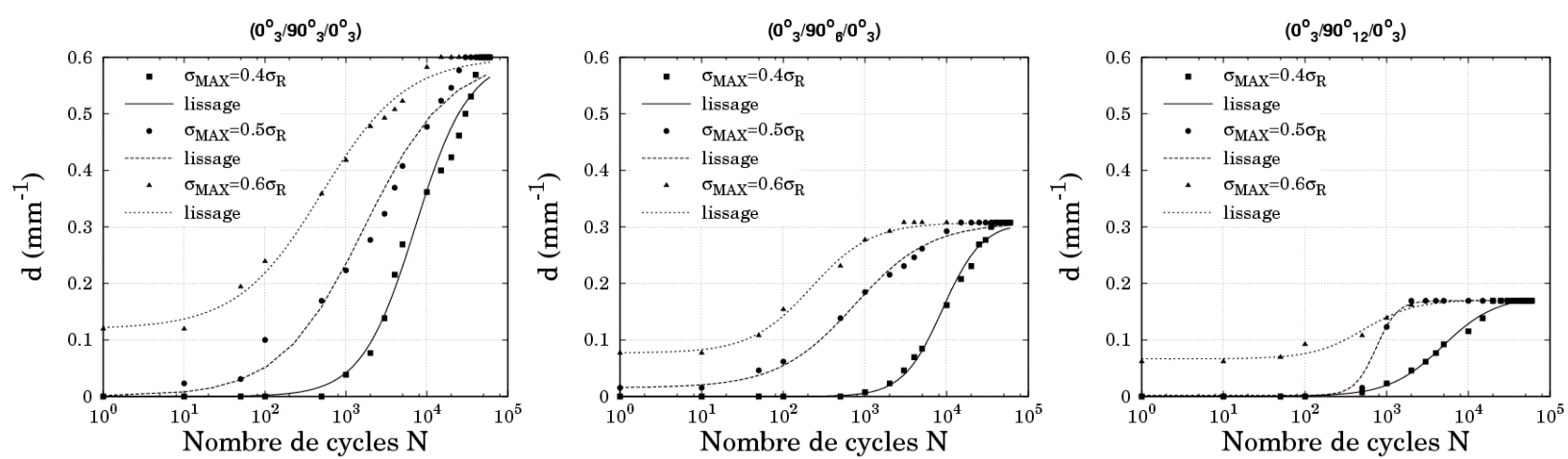

Fig. 5. Évolution de la densité de fissures dans la couche à $90^{\circ}$ et de la rigidité résiduelle pour les séquences $\left(0_{3}^{\circ} / 90_{n=3,6,12}^{\circ} / 0_{3}^{\circ}\right)$ sous chargements cycliques avec $\sigma_{\max }=40 \%, 50 \%$ et $60 \% \sigma_{R}, f=1 \mathrm{~Hz}, R=0,1$.

Fig. 5. Evolution of cracks density in the $90^{\circ}$ layer and the residual stiffness of $\left(0_{3}^{\circ} / 90_{n=3,6,12}^{\circ} / 0_{3}^{\circ}\right)$ laminates under cyclic loadings with $\sigma_{\text {max }}=40 \%, 50 \%$ et $60 \% \sigma_{R}, f=1 \mathrm{~Hz}, R=0.1$.

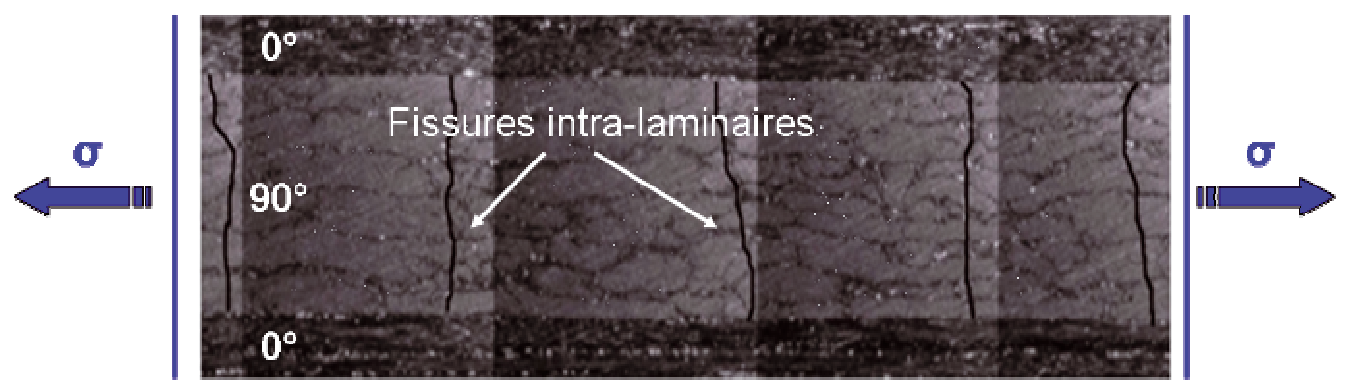

Fig. 6. Réseau de fissures intra-laminaires dans la couche à $90^{\circ} \mathrm{d}^{\prime}$ un stratifié de type $0_{3}^{\circ} / 90_{6}^{\circ} / 0_{3}^{\circ}$ ).

Fig. 6. Network of intra-laminar matrix cracking in the $90^{\circ}$ layer of a $\left(0_{3}^{\circ} / 90_{6}^{\circ} / 0_{3}^{\circ}\right)$ laminates.

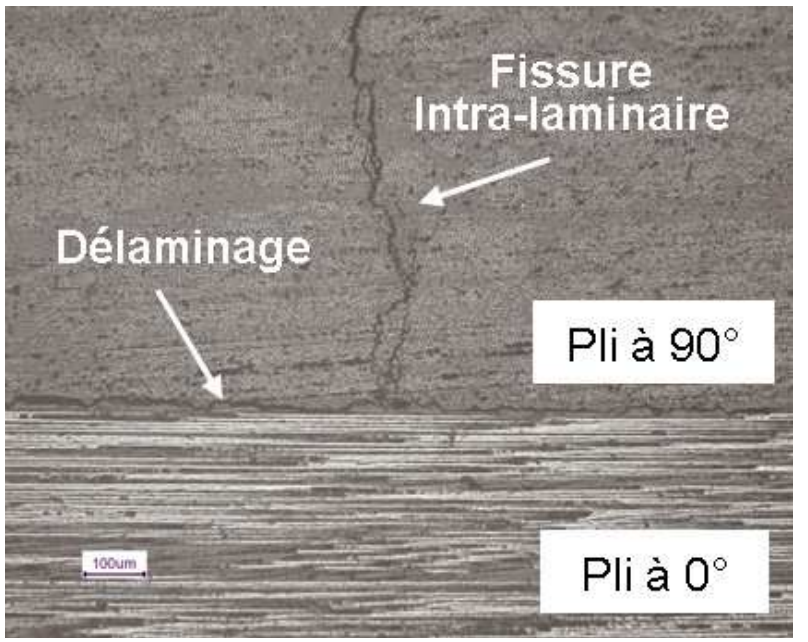

Fig. 7. Apparition de délaminage initié en tête de fissure intra-laminaire

Fig. 7. Onset of delamination at the cracks tip.

réside dans le fait que les fissures sont créées par l'accumulation des cycles de sollicitations et non pas par l'augmentation du chargement. Toutefois, on remarque que le niveau de contrainte appliqué influe sur la cinétique de fissuration. Là encore, la fissuration atteint un état caractéristique à partir duquel le nombre de fissures n'évolue plus (Fig. 5).

Les différents essais réalisés ont permis de mettre en évidence les paramètres qui influencent la fissuration (épaisseur, niveau de chargement) et les caractéristiques de l'endommagement par fissuration intralaminaire. En outre, les fissures sont géométriquement identiques qu'elles proviennent d'un chargement quasi-statique ou cyclique. Elles sont planes, parallèles entre elles et réparties de façon relativement homogène (Fig. 6).

On remarque également l'apparition de délaminage, initié en tête de fissures. Ce délaminage apparaît après que l'état de saturation soit atteint (Fig. 7).

\section{Application : cas d'une éprouvette trouée}

On propose dans cette dernière partie, une application de la méthode d'observation au cas d'une éprouvette trouée. La plaque à trou est en fait une géométrie relativement récurrente dans les structures industrielles comme les panneaux de fuselage par 


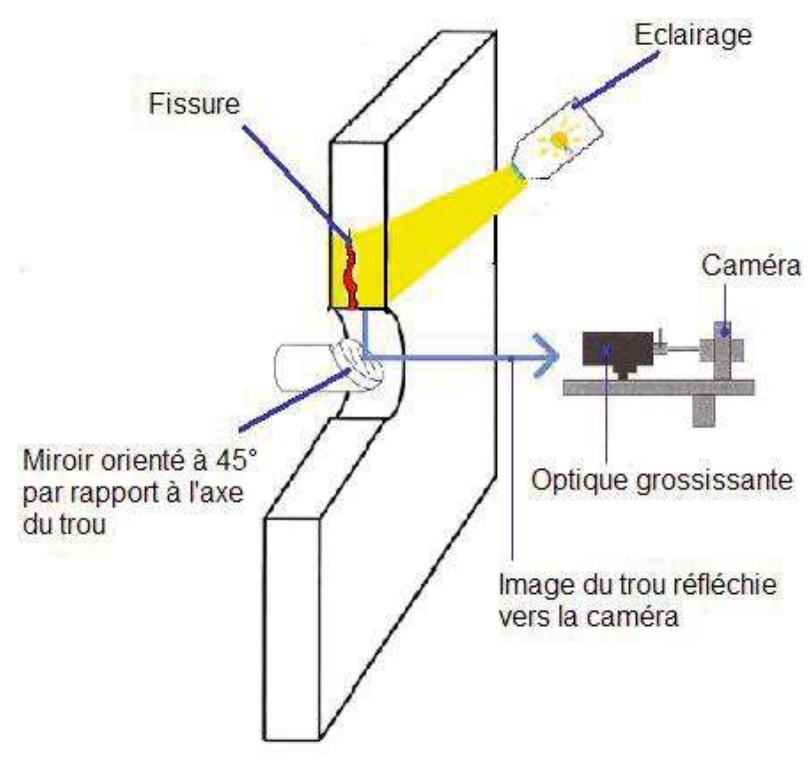

Fig. 8. Schéma du dispositif expérimental pour l'observation de l'endommagement au sein d'un trou.

Fig. 8. Experimental setup for observation of damage in a hole.

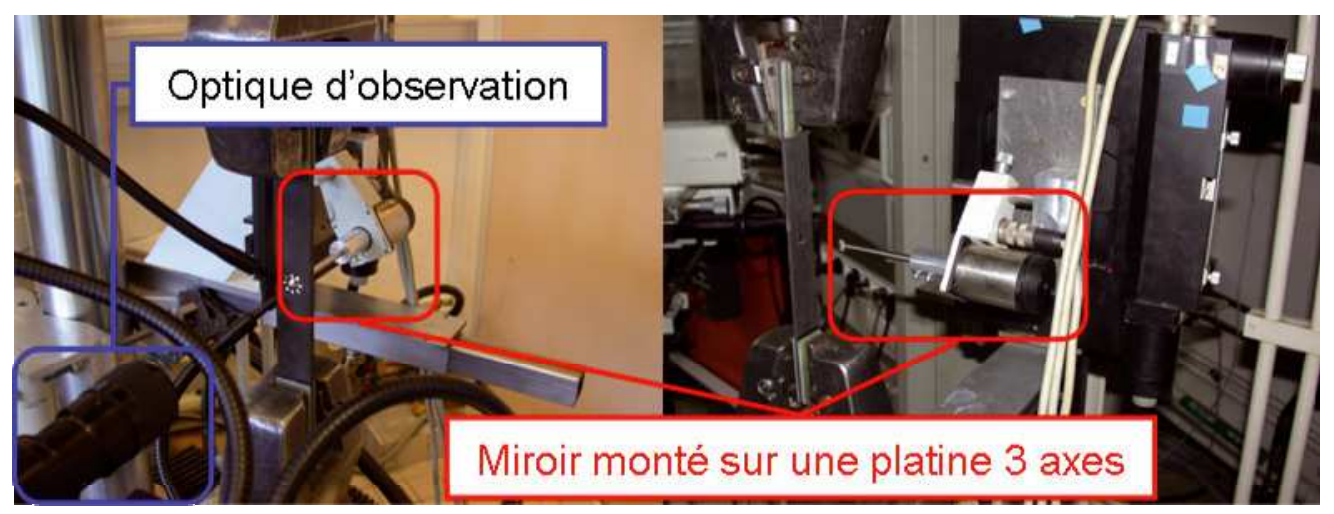

Fig. 9. Montage expérimental pour l'observation d'endommagement au sein d'un trou.

Fig. 9. Experimental setup for observation of damage in a hole.

exemple. Or le trou est un concentrateur de contraintes qui peut favoriser l'apparition d'endommagement. Il apparaît donc important de pouvoir analyser l'évolution de l'endommagement à proximité du trou.

Pour répondre à cette nouvelle problématique, le montage précédent a été équipé d'un miroir orienté à $45^{\circ}$ par rapport à l'axe du trou qui va réfléchir l'image du bord libre du trou vers la caméra (Fig. 8).

Le miroir est monté sur une platine qui permet un déplacement dans les trois directions et une rotation autour de l'axe définit par son support (Fig. 9). Ainsi on peut observer tout le périmètre du trou sur toute l'épaisseur de l'éprouvette.

La procédure à suivre est identique à celle utilisée dans le cas d'une éprouvette droite. Aux différents paliers d'observation on prend plusieurs photographies du bord libre du trou de façon à pouvoir reconstruire une image du trou et analyser les endommagements présents.
La figure 10 représente l'état d'endommagement au bord du trou d'une éprouvette de séquence $\left(0_{3}^{\circ} / 90_{6}^{\circ} / 0_{3}^{\circ}\right)$ sollicitée en fatigue. On constate que la fissuration intra-laminaire est le premier type d'endommagement à apparaître. Là encore, les fissures vont donner naissance à du délaminage.

\section{Conclusion}

Nous avons présenté ici une méthode optique de détection et suivi de l'endommagement in-situ. Cette méthode, simple à mettre en cœuvre permet non seulement de détecter et d'identifier les différents mécanismes d'endommagements présents au sein des matériaux composites. On peut grâce à cette méthode capitaliser des informations à la fois qualitatives et quantitatives. Enfin cette technique permet de mettre en évidence les interactions entre tous les mécanismes d'endommagement. Développée dans le cas 


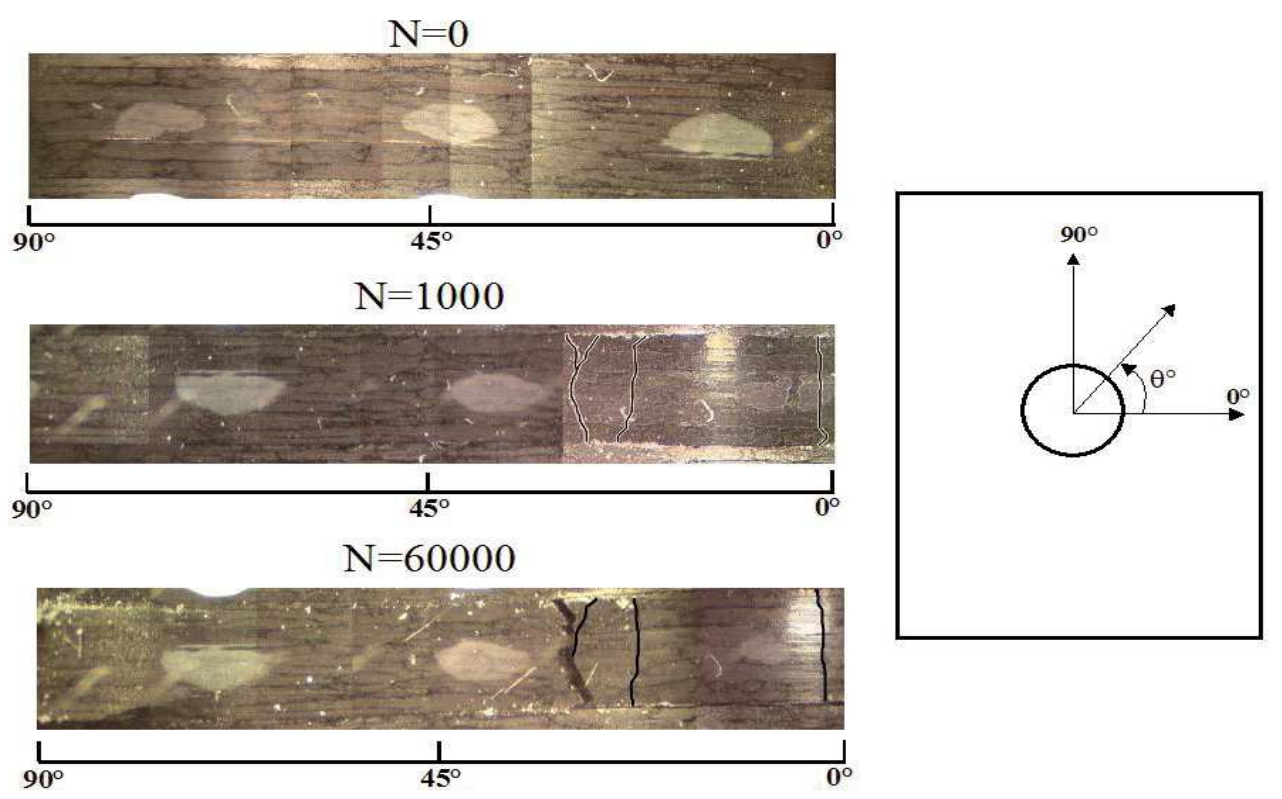

Fig. 10. Évolution de l'endommagement au bord du trou dans une éprouvette trouée de séquence $\left(0_{3}^{\circ} / 90_{6}^{\circ} / 0_{3}^{\circ}\right)$ sollicitée en fatigue, $f=1 \mathrm{hz}, R=0,1, \sigma_{\max }=40 \% \sigma_{R}$.

Fig. 10. Damage evolution in the hole free edge in an open hole laminate $\left(0_{3}^{\circ} / 90_{6}^{\circ} / 0_{3}^{\circ}\right)$ under cyclic loading, $f=1 h z, R=0.1, \sigma_{\max }=40 \% \sigma_{R}$.

d'éprouvettes droites, cette technique a été adaptée et utilisée pour l'observation d'endommagement au sein d'une plaque trouée.

Cette étude a été réalisée dans le contexte $\mathrm{du}$ LAMPC (Laboratoire d'analyse des Matériaux Polymères et Composites), laboratoire commun entre le CETIM de Nantes et le Centre des Matériaux de l'École de Mines de Paris.

\section{Références}

[1] D. Valentin, A.R. Bunsell, A Study of Damage Accumulation in Carbon Fibre Reinforced Epoxy Resin Structures During Mechanical Loading Monitored By Acoustic Emission, Journal of Plastics and Composites, 1 (1982) 314334

[2] P.J. Schilling, B.R. Karedla, A.K. Tatiparthi, M.A. Verges, P.D. Herrington, X-ray computed microtomography of internal damage in fiber reinforced polymer matrix composites, Composites Science and Technology, 65 (2005) 2071-2078
[3] M. Wevers, L. Rippert, J.M. Papy, S. Van Hufel, Processing of transient signals from damage in CFRP composite materials monitored with embedded intensity-modulated fiber optic sensors, NDTEInternational, 39 (2006) 229-235

[4] C. Scarponi, G. Briotti, Ultrasonic technique for the evaluation of delaminations on CFRP, GFRP, KFRP composite materials, Composites: Part B, 31 (2000) 237-243

[5] J. Rossignol, A. Thionnet, Une nouvelle technique de détection des endommagements dans les composites basée sur l'utilisation des micro-ondes et des circuits micro rubans résonants, Comptes Rendus de Mécanique, 334 (2006) 719-724

[6] J.C. Abry, Y.K. Choi, A. Chateauminois, B. Dalloz, G. Giraud, M. Salvia, In situ monitoring of damage in CFRP laminates by means of AC and DC measurements, Composites Sciences and Technology, 61 (2001) 855-864

[7] A. Mian, X. Han, S. Islam, G. Newaz, Fatigue damage detection in graphite/epoxy composites using sonic infrared imaging technique, Composites Sciences and Technology, 64 (2004) 657-666 